\title{
Structure and function of the SEDS:bPBP bacterial cell wall synthesis machinery
}

Megan Sjodt ${ }^{1}$, Kelly Brock², Genevieve Dobihal ${ }^{3}$, Patricia D. A. Rohs ${ }^{3}$, Anna G. Green², Thomas A. Hopf ${ }^{2}$, Alexander J. Meeske ${ }^{3}$, Veerasak Srisuknimit ${ }^{4}$, Daniel Kahne ${ }^{4}$, Suzanne Walker ${ }^{3}$, Debora S. Marks ${ }^{2}$, Thomas G. Bernhardt ${ }^{3}$, David Z. Rudner ${ }^{3}$, Andrew C. Kruse ${ }^{1}$

1Department of Biological Chemistry and Molecular Pharmacology, Harvard Medical School, Boston, MA 02115. ${ }^{2}$ Department of Systems Biology, Harvard Medical School, Boston, MA 02115. ${ }^{3}$ Department of Microbiology and Immunobiology, Harvard Medical School, Boston, MA 02115. ${ }^{4}$ Department of Chemistry and Chemical Biology, Harvard University, Cambridge, MA 02138.

The bacterial cell wall specifies shape and maintains cellular integrity. Inhibiting its synthesis is among the most effective strategies for combating bacterial infections. Recent work has revealed that membrane proteins of the "SEDS" family function as peptidoglycan polymerases, calayzing the concatenation of a lipid II disaccharide pentapeptide headgroup onto a peptidoglycan chain through a glycosyl transfer reaction. In order to understand SEDS protein function, we determined the structure of the prototypical SEDS family member, RodA, using evolutionary covariance fold prediction to enable molecular replacement. The structure reveals a novel ten-pass transmembrane fold with large extracellular loops, as well as a potentially druggable central cavity that is catalytically essential both in vitro and in vivo. In addition, functional and computational studies indicate that RodA and other SEDS proteins directly interact with class B penicillin binding proteins (bPBPs), which catalyze peptidoglycan strand crosslinking. Together, SEDS and bPBPs cooperate to build a cell wall, offering new targets for antibiotic development. 